\title{
微管道中压力驱动液体流动的边界速度滑移
}

\author{
周剑锋，顾伯勤，郡春雷 \\ 南京工业大学机械与动力工程学院, 南京 210009 \\ E-mail: zhoujianfeng@njut.edu.cn \\ 2010-05-21 收稿, 2010-08-11 接受 \\ 国家自然科学基金(10872088)、教育部博士点基金(20070291004, 20093221120009)和南京工业大学学科建设基金资助项目
}

\begin{abstract}
摘要 微通道中气体流动的速度滑移现象已经获得普遍共识. 对于宏观流道中压力驱动的液体 流动, 常常忽略其中的速度滑移. 但当流道的截面尺度减小时, 速度滑移对流动和传热的影响越 来越显著. 基于 Hamaker 均质材料的假设, 建立了液体微团与固体壁面作用力的计算方法. 由近 壁面流体微团的受力分析可知，粗精壁面对近壁面流体微团的作用力可以抵抗来自上层微团的 剪切力, 从而保持近壁面液体微团的静止, 进而提出了液体微团速度滑移的判定准则. 根据发生 滑移的液体微团上的受力平衡关系, 可以确定液体微团所受的壁面摩擦力, 再根据推导得到的壁 面处液体摩擦系数的计算方法, 可确定液体微团的滑移速度量. 研究表明, 微管道中的压力梯度 较大时, 壁面边界处速度滑移可能发生, 若忽略滑移速度则会给管内流量的计算造成误差.
\end{abstract}

关键词

速度滑移

压力驱动 液体分子团

摩擦系数
微泵、微划、微喷以及微混合器等微器件的内部 通道中, 液体的流动通常由压差驱动. 作为微流动系 统传热、传质的主要功能元件, 这些微通道的直径往 往从纳米到微米级不等. 受通道壁面的引力和表面 形貌的影响, 微液体流动特性有别于宏观流道内的 液体流动特性, 壁面边界上的速度滑移已经引起越 来越多的关注.

速度滑移对流体速度和温度分布的影响十分显 著, 而经典流体动力学则基于壁面边界处流体速度 与壁面速度保持严格一致的基本假设. 研究表明，一 旦边界处的流体剪切速率超过临界值, 就会出现速 度的不连续和滑移. 现有关于速度滑移的研究主要 集中于微通道内的气体流动, 这主要是由于气体的 黏度相对较小而分子间距较大, 更容易发生速度滑 移. 基于 Maxwell一阶速度滑移表达式, 文献[1]将二 阶速度滑移边界条件引人 Navier-Stocks 方程中, 以 扩大 Navier-Stocks 方程的应用范围. Sparrow 等人 ${ }^{[2]}$ 发现, 微型圆管道中的曳力系数和传热系数均小于 宏观管道. 文献[3]在分析 Graetz 问题时考虑了速度 滑移和温度阶跃，这两个条件亦被用于求解微圆形
管道 ${ }^{[4]}$ 和矩形管道 ${ }^{[5]}$ 中不可压缩气体流动的 NavierStocks 方程和能量方程. 文献[6]的研究发现, 速度滑 移量在很大程度上取决于流体温度和壁面引力.

受微通道的尺度限制, 一些流动参数的试验测 试十分复杂且困难, 速度滑移量尚不能直接测量. 分 子动力学模拟方法被广泛应用于纳米微通道中气体 流动的速度滑移研究. 文献[7]采用球分子模型研究 了液态氩薄膜的速度滑移, 文献[8]也采用分子动力 学方法研究了超薄润滑膜的界面滑移特性. 文献 [9] 采用非平衡分子动力学方法研究了纳米通道中的 Couette 流动, 发现了诸如滑移、无滑移以及负滑移 等现象. 文献[10]的研究发现, 纳米级薄膜中的速度 滑移在较小的剪切速率条件下即可发生. 后来的研 究发现, 纳米级薄膜中的分层和滑移现象始终存 在 $^{[11]}$. 文献[12 14]采用 Burnett 模拟方法研究了 Couette 和 Poiseuille 流的流动和传热特性. 文献[15] 在研究压力驱动不可压缩牛顿流体的瞬态流动时采 用了 Navier 速度滑移边界条件. 此外, 文献[16 19] 亦对速度滑移和移动边界从不同角度进行了研究. 然而, 当管道界面的尺寸为微米级时, 由于分子数量 
十分庞大, 分子动力学模拟方法已不适用于速度滑 移问题的研究.

考虑到液体的黏度要大于气体, 在分析微槽道 或微管道中液体的流动特性时, 往往忽略其中的速 度滑移. 但对于具有纳米级或微米级通道的高精度 流量传感器而言, 速度滑移会在不同程度上影响流 体流动. 本文着重对微管道中液体流动的速度滑移 现象作理论分析，建立近壁面流体层速度滑移的判 定准则, 并提出速度滑移量的计算方法.

\section{1 圆形微管道中压力驱动的液体流动模型}

图 1 为圆形管道的轴截面示意图, 其中 $p_{1}$ 和 $p_{2}$ 分别为进口和出口压力, $r$ 和 $z$ 分别为径向和轴向坐 标, $v(r)$ 为液体的速度分布方程, $\Delta v$ 为速度滑移量.

与流道半径相比, 内壁面的粗糙度水平不可忽 略. 假设管道中为牛顿流体的层流流动, 根据牛顿剪 切定律, $v(r)$ 可表示为

$$
v(r)=-\frac{\Delta p}{L} \frac{r^{2}}{4 \mu}+\frac{C_{1}}{\mu} \ln r+C_{2},
$$

其中 $\Delta p=p_{1}-p_{2}, \mu$ 为流体的动力黏度, $L$ 为管道长度. $C_{1}$ 和 $C_{2}$ 为待定常数, 可根据边界条件进行求解, 即: 当 $r=0$ 时剪切应力为 0 , 而在壁面边界处, $v(R)=\Delta v$. 于 是 $v(r)$ 可表示为

$$
v(r)=\frac{\Delta p}{4 \mu L}\left(-r^{2}+R^{2}\right)+\Delta v,
$$

其中 $R$ 为管道内半径. 剪切应力为 $r$ 的函数, 即

$$
\tau(r)=-\frac{\Delta p}{2 L} r .
$$

式(3)表明流体内部的剪切应力是沿 $z$ 方向的压 力梯度和半径 $r$ 的函数. 速度滑移量 $\Delta v$ 对剪切应力 $\tau(r)$ 的分布没有影响.

\section{2 固体壁面与液体作用力的计算}

液体的黏性主要由分子间的内聚力和附着力引 起，黏性使得液体能黏着在固体壁面上，然而液体黏 性仅当承受剪切时才能体现. 由于分子间的作用势, 液体分子间的作用力始终存在. 液体分子的运动具 有高度随机性, 因此无法通过计算液体分子与固体 壁面分子间的作用力来确定液体和固体的作用力.

\section{1 分子团的作用力}

为了采用连续方法解决微观物质世界的离散问 题, Hamaker 提出了 3 个假设, 即: (1) 离散模型可加 性假设，即任何两个物体之间的作用力由构成该两 个物体的原子对之间的作用力累加求和得到(图 2 中, $M_{1}$ 内的每个分子均和 $M_{2}$ 中的每个分子组成分子对); (2) 连续介质假设, 即任何物体由数值密度为 $Q$ 的微 元体积 $\mathrm{d} v$ 连续构成; (3) 均质介质材料假设, 即任何 物体, 数字密度 $Q$ 和引力、斥力常数不变. 这 3 个假 设已经得到了很好的发展和应用 ${ }^{[20,21]}$. 根据这 3 个假 设, 可以认为连续液体是由若干具有一定形状和分 子数密度的微团组成, 每个微团均由同种液体分子 构成. 如图 2 所示的两个分子团 $\mathrm{M}_{1}$ 和 $\mathrm{M}_{2}, V_{1}$ 和 $V_{2}$ 分 别为这两个微才的体积, 这两个微团由若干液体分 子组成, 假设这些分子都处于静止状态. 这样, 原本 计算随机运动的分子间的作用力就转化为计算静止 的分子团间的作用力，问题得以简化.

采用 Lennard-Jones 12-6 势能模型计算分子对间 的作用力, 该模型可表示为

$$
U=4 \varepsilon\left[\left(\frac{\sigma}{l}\right)^{12}-\left(\frac{\sigma}{l}\right)^{6}\right] .
$$

在某一微团内部，不同分子间的作用力属于内 力, 因此仅考虑分子团 $\mathrm{M}_{1}$ 内部的分子与 $\mathrm{M}_{2}$ 内部的分 子间的作用力. 由 Lennard-Jones 势引起的分子间的

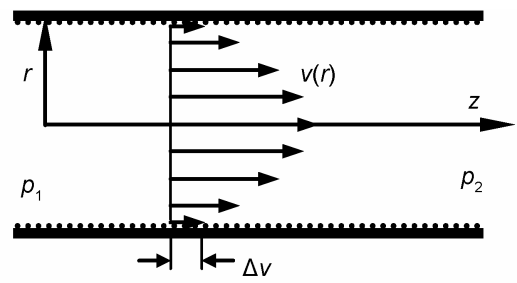

图 1 圆形管道中的压力驱动流体流动模型

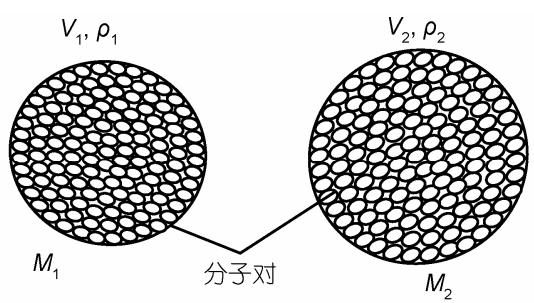

图 2 液体分子团模型 
作用力可按式(5)计算.

$$
f(l)=\frac{A}{l^{13}}-\frac{B}{l^{7}}=f_{1}(l)-f_{2}(l),
$$

其中 $f_{1}(l)$ 为斥力部分, $f_{2}(l)$ 为引力部分, $A$ 和 $B$ 为常数. 通过积分 $f(l)$ 可得分子团 $\mathbf{M}_{1}$ 和 $\mathbf{M}_{2}$ 间的作用力, 即

$$
F=\rho_{1} \rho_{2} \iint_{V_{2} V_{1}}\left[f_{1}(l)-f_{2}(l)\right] \mathrm{d} v_{1} \mathrm{~d} v_{2},
$$

其中 $\rho_{1}$ 和 $\rho_{2}$ 分别为分子团 $\mathrm{M}_{1}$ 和 $\mathrm{M}_{2}$ 的分子数密度.

式(6)的积分运算十分复杂, 到目前为止, 仅推 导得到了球形边界的分子团的作用力解析表达 式 ${ }^{[20 ~ 22]}$. 本文采用矩形边界的分子团模型, 因其积 分过程过于复杂而无法得到解析计算方程, 故采用 数值积分法来计算分子团间的作用力.

\section{2 分子团几何尺寸的确定}

虽然分子团的尺寸要远大于分子的直径, 其仍 然远小于微管道的半径和长度. 对于近壁面液体层, 采用矩形微团较为合适, 故从该液体层中取出宽度 为 $w_{1}$ 的液体微团, 而从相应的壁面上取出宽度为 $w_{2}$ 固体微团, 如图 3 所示, $h_{1}$ 和 $h_{2}$ 分别为这两个微团的 高度, $\Delta$ 为微团间距. 这些几何参数均要通过分析作 用力的有效范围才能确定, 分析时分子团的厚度取 为 $1 \times 10^{-8} \mathrm{~m}$.

分析时采用的流体为液体氩( $\mathrm{Ar}$ ), 而固体分子为 假想分子 $(\mathrm{X})$, 这两种分子均采用形如式(4)的势能方 程. 分子 $\mathrm{Ar}$ 和 $\mathrm{X}$ 的物理参数列于表 1 中.

液体和固体分子间的碰撞直径 $\sigma_{12}$ 表示为

$$
\sigma_{12}=\sigma_{1} \frac{d_{1}+d_{2}}{2 d_{1}} \text {. }
$$

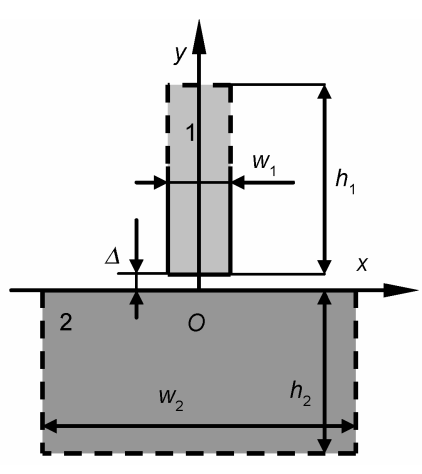

图 3 液体和固体的矩形分子团模型
表 1 分子的物理参数

\begin{tabular}{cccc}
\hline & 下脚标 & 分子质量 $m / \mathrm{kg}$ & 势能常数 $\varepsilon / \mathrm{J} \cdot \mathrm{K}^{-1}$ \\
\hline $\mathrm{Ar}$ & 1 & $6.633 \times 10^{-26}$ & $1.67 \times 10^{-21}$ \\
$\mathrm{X}$ & 2 & - & $8.35 \times 10^{-21}$ \\
\hline \hline & 碰撞直径 $\sigma / \mathrm{m}$ & 分子间距 $d / \mathrm{m}$ & 分子数密度 $\rho / \mathrm{m}^{-3}$ \\
$\mathrm{Ar}$ & $3.47 \times 10^{-10}$ & $3.71 \times 10^{-10}$ & $1.94 \times 10^{28}$ \\
$\mathrm{X}$ & $2.75 \times 10^{-10}$ & $2.17 \times 10^{-11}$ & $7.80 \times 10^{28}$ \\
\hline
\end{tabular}

两个分子团均关于坐标轴 $y$ 对称，故分子团间的 作用力沿坐标轴 $x$ 方向的分量为 0 . 分子团间隙 $\Delta$ 对 作用力的影响十分显著, 这可从图 4 看出, 其中 $F_{y}$ 是沿 $y$ 方向的分力. 当 $\Delta$ 从 $1 \times 10^{-14} \mathrm{~m}$ 增大到 $1 \times 10^{-12} \mathrm{~m}$ 时, 由于引力和斥力的交互作用, $F_{y}$ 逐渐减小; 当 $\Delta$ 超过 $1 \times 10^{-12} \mathrm{~m}$ 时, $F_{y}$ 迅速减小. 此例中, $h_{1}=h_{2}=1 \times 10^{-8}$ $\mathrm{m}, w_{1}=w_{2}=1 \times 10^{-8} \mathrm{~m}$. 由于引力比斥力大得多, 其合 力表现为引力, 使得分子团互相吸引. 分析表明, $\Delta$ 取 $1 \times 10^{-13} \mathrm{~m}$ 较为合适.

$F_{y}$ 随 $w_{1}$ 的变化规律如图 5 所示, 此时 $h_{1}=1 \times 10^{-8} \mathrm{~m}$, $w_{2}=1 \times 10^{-8} \mathrm{~m}, h_{2}=1 \times 10^{-8} \mathrm{~m}, \Delta=1 \times 10^{-13} \mathrm{~m}$. 当 $w_{1}$ 从 $1 \times$ $10^{-8} \mathrm{~m}$ 增大到 $2 \times 10^{-8} \mathrm{~m}$ 时, $F_{y}$ 增大, 而当 $w_{1}>2 \times 10^{-8} \mathrm{~m}$ 时, $F_{y}$ 几乎不变.

同理，当 $w_{1}=1 \times 10^{-8} \mathrm{~m}$ 时，将 $w_{2}$ 从 $1 \times 10^{-8} \mathrm{~m}$ 增大 至 $1 \times 10^{-7} \mathrm{~m}$, 发现能促使 $F_{y}$ 增大的 $w_{2}$ 的范围为 $1 \times 10^{-8} \sim 2 \times 10^{-8} \mathrm{~m}$. 因此, 对于给定的液体分子团, 相 应的固体分子团的有效宽度可取 2 倍于液体分子团 的宽度.

图 6 表明了 $F_{y}$ 随 $h_{1}$ 的变化规律. 由于分子作用 势的有效作用范围仅数倍于碰撞直径 $\sigma$, 因此一旦 $h_{1}>4 \times 10^{-9} \mathrm{~m}, h_{1}$ 的增加不再引起 $F_{y}$ 的显著增加. 由 $h_{2}$ 的增大引起的 $F_{y}$ 的变化规律与图 6 类似. 于是, 取

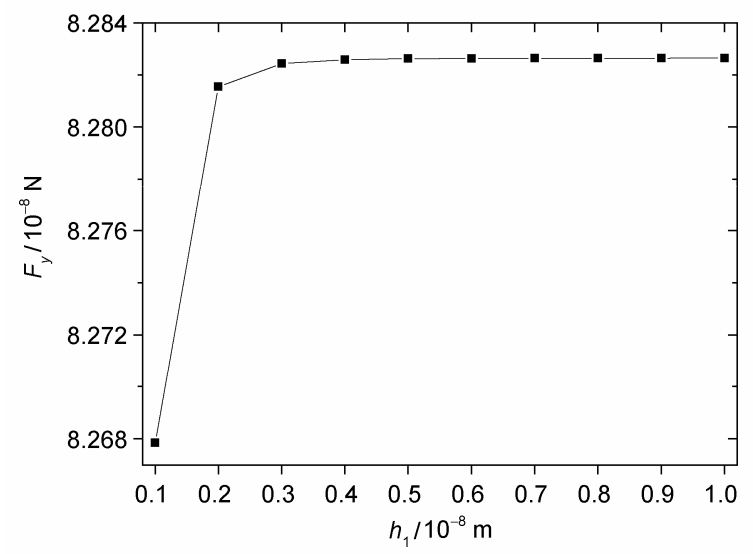

图 $4 F_{y}$ 随 $\Delta$ 的变化 


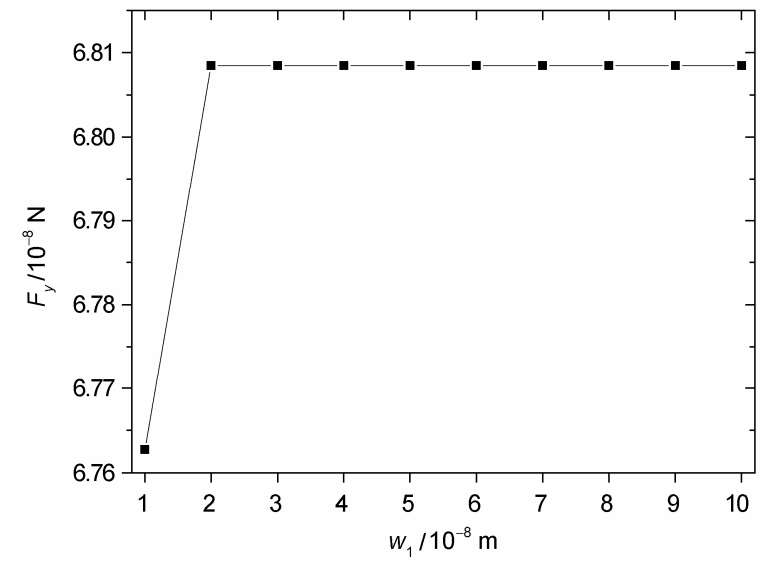

图 $5 \quad F_{y}$ 随 $w_{1}$ 的变化

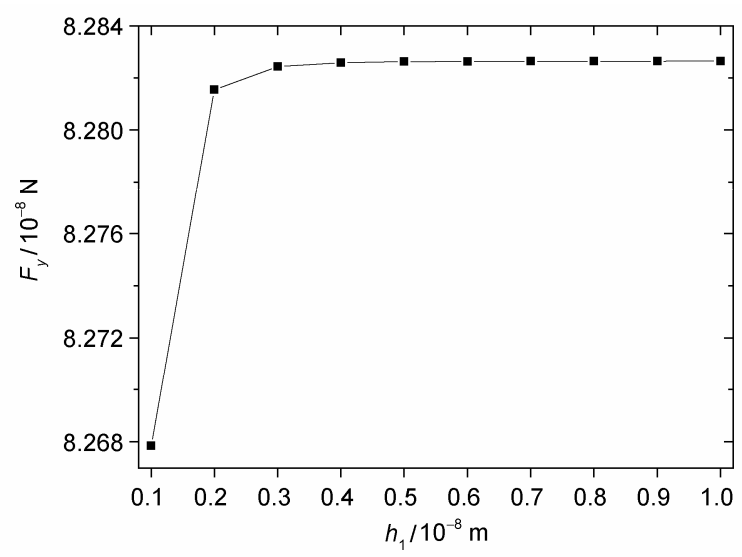

图 $6 F_{y}$ 随 $h_{1}$ 的变化

$h_{1}=1 \times 10^{-8} \mathrm{~m}$ 和 $h_{2}=1 \times 10^{-8} \mathrm{~m}$ 可满足分子团最大作用 力的计算精度要求.

由于固体分子的作用势大于液体分子, 两个液 体微团(图 7)间的作用力略小于液体微团与固体微团 之间的作用力, 如图 8. 图 8 说明液体分子团间的作 用力不会无限增大, 因此, 对于近壁面的流体微团, 若固体壁面对其吸引力大于上层微团的吸引力, 该 微团就可能保持静止.

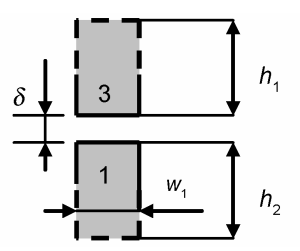

图 7 相邻液体分子团的模型

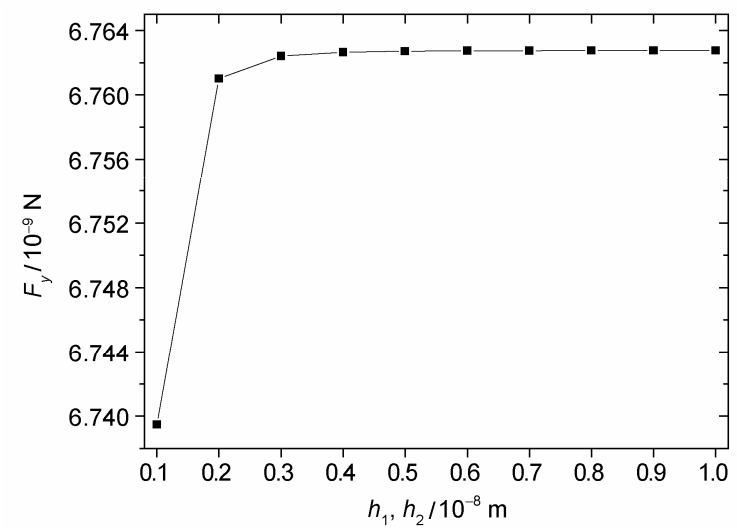

图 $8 F_{y}$ 随 $h_{1}\left(h_{2}\right)$ 的变化

\section{3 速度滑移的发生条件}

\section{1 近壁面流体层内的剪切应力}

图 9 表示两个相邻的流体微团. 由于剪切应力和 速度梯度的作用, 经过一个时间步后, 这两个微团 $a-b-c-d$ 和 $e-f-g-h$ 将分别变形成为 $a^{\prime}-b^{\prime}-c^{\prime}-d^{\prime}$ 和 $e^{\prime}-f^{\prime}-g^{\prime}-h^{\prime}$. 在此过程中, 微团间作用力的分力 $F_{x}$ 和 $F_{y}$ 将由于微团发生角变形而变化, 结果如图 10 所示.

由图 10 可知, 当两个微团发生相对运动时, 分力 $F_{x}$ 和 $F_{y}$ 不是常数且与速度无关. 从 $F_{x}$ 的变化规律看, $F_{x}$ 与速度无关, 故不能视其为两个微团间的剪切力. 因此不能采用 2.1 节提出的方法计算分子团的剪切力.

按照式(3)计算近壁面流体层间的剪切应力, 结 果如图 11 所示, 计算参数为 $R=1.0 \times 10^{-3} \mathrm{~m}, L=0.1 \mathrm{~m}$, $p_{1}=1.1 \times 10^{5} \mathrm{~Pa}, p_{2}=1.0 \times 10^{5} \mathrm{~Pa}, \Delta r=R-r$. 剪切应力 $\tau$ 沿 半径方向呈线性分布, 且最大剪切应力位于壁面边 界上.

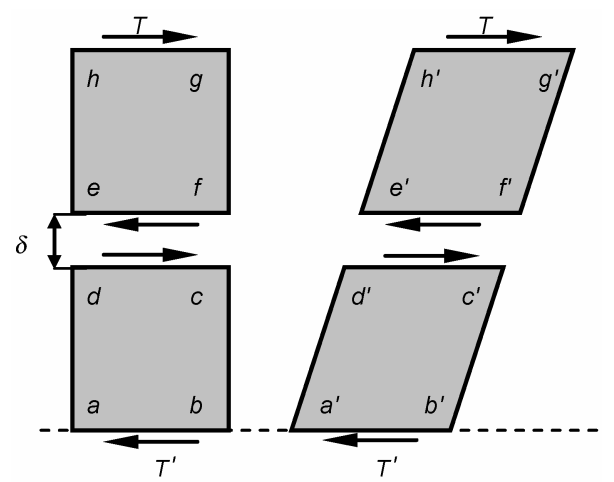

图 9 相邻液体微团的剪切模型 


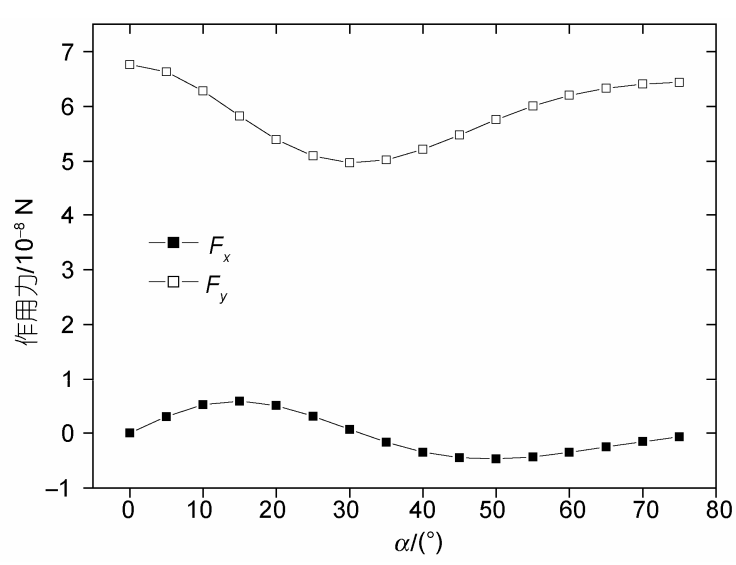

图 $10 F_{x}$ 和 $F_{y}$ 随角变形 $\alpha$ 的变化

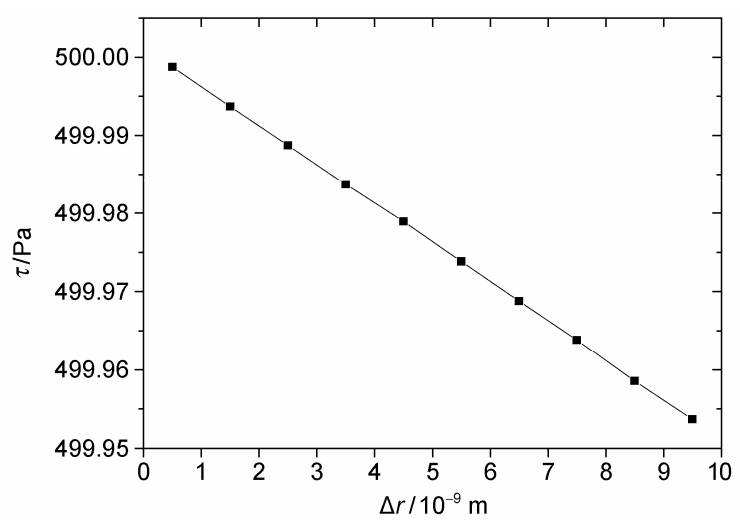

图 $11 \Delta r$ 剪切应力 $\tau$ 随 $\Delta r$ 的变化

\section{2 近壁面液体微团的受力分析}

真实的固体壁面是粗糙的, 峰谷随机分布. 从纳 米尺度上观察, 固体表面由无数固体分子组成, 而固 体分子又直接暴露于液体分子中. 作用于液体微团 上的力的方向可以沿着图 12(a)所示的 $\boldsymbol{n}$ 方向, 亦可 以为图 12(b) 所示的 $\boldsymbol{n}^{\prime}$ 方向 ( $\theta$ 相对较小时).

如果固体壁面作用在近壁面液体微团 2 上的作 用力的方向与上层液体分子团 3 作用在该分子团上 的剪切力的方向垂直, 则分子团 2 不能保持静止状态. 在剪切力的作用下, 分子团 2 将发生偏转, 这样壁面 对其的作用力方向也发生改变. 于是, 壁面作用力就 可以提供一个分力与剪切力抗衡, 分子团 2 则有可能 保持静止.

对粗粘的固体壁面进行简化, 如图 13 所示, 由 若干连续的倾斜面组合代替随机部分的粗鋉元. 倾 角 $\eta$ 与液体分子团的倾斜程度相关，通过改变倾斜面
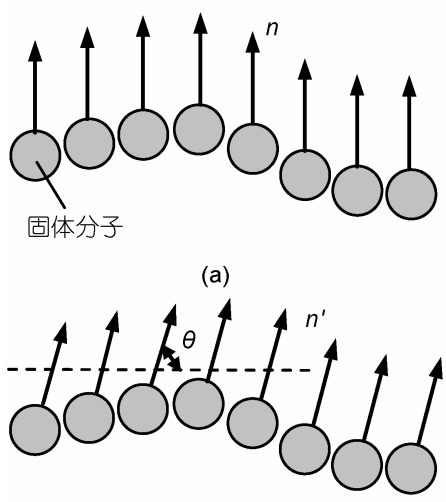

(b)

图 12 固体壁面作用力的方向

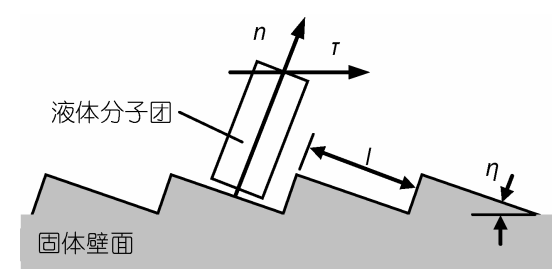

图 13 简化的粗粕固体表面和近壁面的液体分子团

长度 $l$ 可满足不同粗鋉度的要求.

近壁面液体微团的受力情况如图 14 所示. $F_{y}$ 为 固体微团对流体微团的作用力, $F_{s}$ 为剪切力, $F_{u}$ 为上 层液体微团的作用力. 若这 3 个力的合力为 0 , 则微 团静止.

保持液体分子团的上表面面积不变, 分子团的 变形过程中, $F_{y}$ 和 $F_{u}$ 都将发生改变. 随着 $F_{\tau}$ 的增大, $\theta$ 相应减小, 合力 $F_{1}$ 的变化情况却未知. 对于固体表 面, 液体微团的宽度由 $w_{1}$ 变化到 $w_{1}^{\prime}$, 且 $w_{1}^{\prime}=w_{1} \sin \theta$. 由于 $F_{u}$ 是 $\theta$ 的复杂函数, 即 $\theta=\arctan \left(F_{u} / F_{s}\right)$, 因此按 图 15 所示的流程求解 $\theta$.

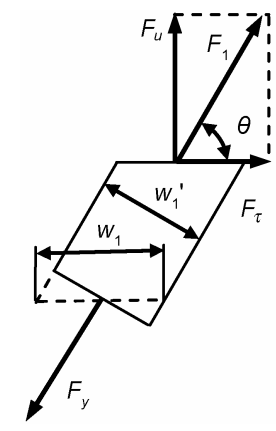

图 14 近壁面液体微团上的作用力 


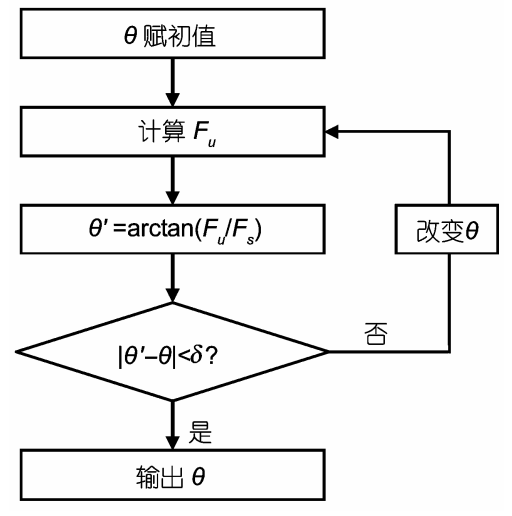

图 $15 \theta$ 的计算流程

\section{3 液体分子团的滑移}

影响壁面边界处剪切应力的操作条件是压力梯 度 $\Delta p / L . F_{1}$ 和 $F_{y}$ 随压力梯度的变化规律如图 16 所示, 其中 $k=\Delta p / 2 L$.

当 $k$ 增大时, $F_{1}$ 和 $F_{y}$ 均减小, $F_{y}$ 的减小幅度要远 大于 $F_{1}$. 当 $k>8.7 \times 10^{3} \mathrm{~Pa} \cdot \mathrm{m}^{-1}$ 时, $F_{y}$ 小于 $F_{1}$, 这意味着 固体壁面不再能拖曳液体分子团，此时滑移发生.

除了操作参数以外, 分子势能函数中包含的参 数亦对液体分子团的滑移有影响. 例如, 当液体分子 的势能常数 $\varepsilon_{1}$ 增加时, $\varepsilon_{1}$ 和 $\varepsilon_{2}$ 的差值减小, 引起液体 微团滑移的临界 $k$ 值就小于 $8.7 \times 10^{3} \mathrm{~Pa} \cdot \mathrm{m}^{-1}$.

\section{4 速度滑移量的计算}

\section{1 滑移液体分子团上的受力分析}

一旦液体分子团发生了滑移，则认为作用于液 体分子团上的力 $F_{y}$ 的方向垂直于壁面，不再考虑壁

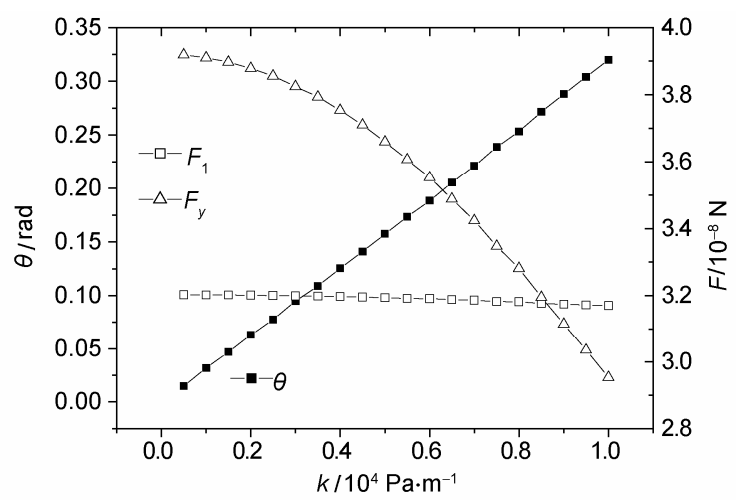

图 $16 F_{1}$ 和 $F_{y}$ 随 $k$ 的变化规律
面的粗粘度影响. 此外, 来自壁面的摩擦力 $F_{f}$ 将作用 到液体分子团上, 如图 17 所示, $F_{p 1}$ 和 $F_{p 2}$ 分别为液体 分子团左右两侧的、由压力引起的作用力.

图 16上所示的力必须满足式(8)和(9)表示的平衡 关系.

$$
\begin{gathered}
F_{u}=F_{d}, \\
F_{p 1}-F_{p 2}+F_{\tau}=F_{f} .
\end{gathered}
$$

\section{2 液体在固体边界上的摩擦系数}

对于某一运动分子, 其运动总是要受到周围分 子作用合力的阻止，这种阻滞效应可以定义为摩擦 力, 且该摩擦力的大小与分子运动速度成正比，可表 示为 ${ }^{[23]}$

$$
F(t)=-\beta m v+G(t),
$$

其中 $G(t)$ 是随机波动力, $\beta$ 是摩擦系数, $m$ 和 $v$ 分别是 分子的质量和速度.

Rice 等人 ${ }^{[24]}$ 给出了球坐标系下 $\beta$ 的表达式:

$$
\beta=\left[\frac{\rho}{3 m} \int_{0}^{2 \pi} \int_{0}^{\pi} \int_{0}^{\infty} r^{2}\left(\frac{\partial^{2} U}{\partial r^{2}}+\frac{2}{r} \frac{\partial U}{\partial r}\right) g(r) \sin \theta \mathrm{d} r \mathrm{~d} \theta \mathrm{d} \phi\right]^{1 / 2},
$$

其中, $\rho$ 是液体分子的分子数密度, $g(r)$ 是分子的平衡 径向分布方程，即

$$
g(r)=\exp \left[-\frac{U}{k_{b} T}\right],
$$

其中 $T$ 是绝对温度, $k_{b}$ 是 Boltzmann 常数.

一般来说，固体分子会影响其周围的液体分子 的分布，因此提出以下两个假设来忽略这种影响：(1) 液体分子的分布不受固体分子的影响，且液体分子 间的作用力亦不受固体分子的影响; (2) 固体分子均 匀分布且其平衡径向方程等于 1.

图 18 所示为一个球形控制体, 要确定固体边界

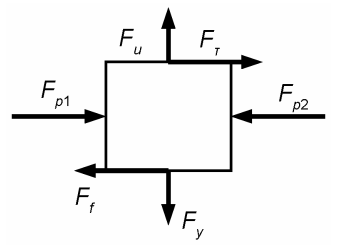

图 17 滑移条件下作用于液体微团上的力 


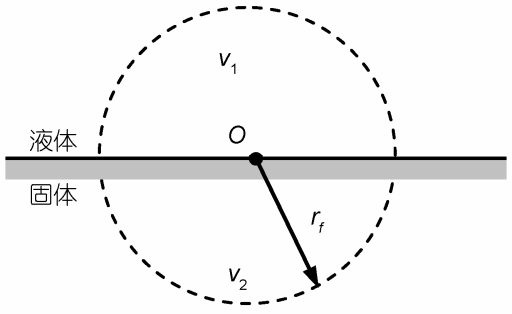

图 18 用于计算摩擦系数的球形控制体

处, 即该控制体中心位置的摩擦系数 $\beta$. 对液体分子 的阻滞效应同时来自于控制体内的固体和液体分子. 对于上半部分球体 $v_{1}, \beta_{1}$ 可由式(13)计算.

$$
\beta_{1}=\left[\frac{\rho}{3 m} \int_{0}^{2 \pi} \int_{0}^{\pi / 2} \int_{0}^{\infty} r^{2}\left(\frac{\partial^{2} U}{\partial r^{2}}+\frac{2}{r} \frac{\partial U}{\partial r}\right) g(r) \sin \theta \mathrm{d} r \mathrm{~d} \theta \mathrm{d} \phi\right]^{1 / 2} .
$$

对于下半部分球体 $v_{2}, \beta_{2}$ 可根据式(14)计算 ${ }^{[25]}$

$$
\beta_{2}=\left[\frac{1}{3 m}\left(f_{2}-f_{1}\right)\right]^{1 / 2},
$$

其中

$$
\begin{aligned}
& f_{1}=\rho \int_{0}^{2 \pi} \int_{0}^{\pi / 2} \int_{0}^{\infty} r^{2}\left(\frac{\partial^{2} U}{\partial r^{2}}+\frac{2}{r} \frac{\partial U}{\partial r}\right) g(r) \sin \theta \mathrm{d} r \mathrm{~d} \theta \mathrm{d} \phi \\
& f_{2}=\rho_{s} \int_{0}^{2 \pi / 2} \int_{0}^{\pi} \int_{0}^{\infty} r^{2}\left(\frac{\partial^{2} U_{s}}{\partial r^{2}}+\frac{2}{r} \frac{\partial U_{s}}{\partial r}\right) \sin \theta \mathrm{d} r \mathrm{~d} \theta \mathrm{d} \phi
\end{aligned}
$$

其中 $\rho_{s}$ 和 $U_{s}$ 分别为固体分子的分子数密度和势能方 程. 于是液体分子的摩擦系数 $\beta$ 可表示为

$$
\beta=\sqrt{\beta_{1}^{2}+\beta_{2}^{2}} .
$$

方程(13), (15)和(16)可采用数值积分法进行求解. 采用表 1 所列的参数, 计算得到壁面边界处的液体分 子的摩擦系数为 $1.961 \times 10^{13} \mathrm{~s}^{-1}$. 于是得到了壁面边 界处的液体分子团的速度滑移量 $\Delta v$, 其随着 $\Delta p$ 的增大 而增大, 如图 19 所示.

虽然速度滑移量 $\Delta v$ 较小, 但其确实存在. 当管道 的半径变小时, 由速度滑移引起的流量误差也逐渐 增大. 例如, 当 $\Delta p=0.5 \times 10^{5} \mathrm{~Pa}, R=6.25 \times 10^{-5}$ 时, 由 $\Delta v$ 引 起的流量差约为 $0.37 \%$. 如果管道内径变得更小, 误 差将变得更大.

研究发现, 速度滑移量与管道的压力差成正比, 这与文献[16]和[17]的研究结果一致. 目前尚未有液

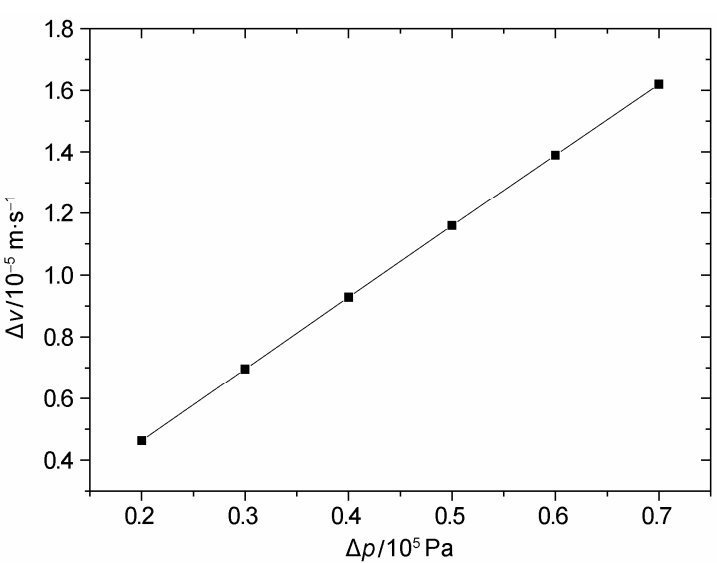

图 19 速度滑移量 $\Delta v$ 与压差 $\Delta p$ 的关系

体边界速度滑移的定量计算方法, 故本文的理论分 析结果有待试验验证. 为了描述固体边界处的液体 滑移特性, Navier 推导了一个通用边界条件, 即与固 体表面相切的速度滑移分量, 如式(18)所示, 其与液 固界面的剪切应力成正比.

$$
\Delta v=-l^{*} \frac{\tau(R)}{\mu},
$$

其中 $l^{*}$ 是速度滑移长度, 可通过试验测量流量 $Q$ 来 确定 ${ }^{[15]}$.

$$
\frac{2 \mu L}{\pi R^{3} \Delta p} Q=l^{*}+\frac{R}{4} .
$$

如果 $l^{*}=0$, 则不存在速度滑移. 如果 $l^{*}>0$, 则在 壁面边界处存在速度滑移，且测得的实际流量要大 于无滑移时的流量. 后续的研究将采用试验方法研 究微管道内的液体流动速度滑移规律.

采用 Hamaker 假设, 忽略了分子随机运动对速 度滑移的影响, 也未考虑温度的影响. 此外, 根据动 力学理论, 速度滑移主要受流体分子与固体壁面碰 撞过程(可定义为镜面反射和漫反射)的影响，壁面粗 䊁度的影响也未考虑.

\section{5 结论}

微管道内液体的速度滑移对液体的流动和传热 有显著影响, 速度滑移与壁面特性和壁面边界处的 剪切应力直接相关.

根据近壁面液体分子团的受力情况提出了速度 滑移条件. 假设管道内的液体分子均匀分布，不考虑 分子随机运动的影响, 采用数值积分法计算液体分 
子团与固体壁面的作用力. 通过分析分子作用力的 有效范围确定了液体分子团的几何参数. 对粗粘的 固体壁面进行了简化，当壁面能够提供足够大作用 力以抵抗上层液体的剪切力时, 可保持近壁面微团 的静止状态. 液体及固体分子的作用势参数、液体内
部的剪切应力均会影响速度滑移的发生.

发生速度滑移的壁面微团要承受来自于壁面的 摩擦力, 该摩擦力与液体微团的速度成正比. 采用推 导得到的液体分子的摩擦系数计算壁面摩擦力, 可 确定速度滑移量.

\section{参考文献}

1 谢翀, 㚞菁. Navier-Stocks 方程二阶速度滑移边界条件的检验. 力学学报, 2007, 39: 1-6

2 Sparrow E M, Lin S H. Laminar heat transfer in tubes under slip-flow conditions. ASME J Heat Transfer, 1962, 84: 363-369

3 Barron R F, Wang X M, Ameel T A, et al. The Graetz problem extended to slip-flow. Int J Heat Mass Transfer, 1997, 40: 1817-1823

4 朱峋, 辛明道. 滑移流区内微环缝槽道中的层流流动与换热. 工程热物理学报, 2001, 22: 203-206

5 肖睿, 辛明道, 朱恂. 微矩形槽道内的气体滑移流动和传热分析. 重庆大学学报(自然科学版), 2004, 24: 99-103

6 徐超, 何雅玲, 王勇. 纳米通道滑移流动的分子动力学模拟研究. 工程热物理学报, 2005, 26: 912-914

7 Thompson P A, Robbins M O. Shear flow near solids: Epitaxial order and flow boundary conditions. Phys Rev A, 1990, 41: 6830—6837

8 王慧, 胡元中, 郭炎. 超薄润滑膜界面滑移现象的分子动力学研究. 清华大学学报(自然科学版), 2000, 40: 107-110

9 曹炳阳, 陈民, 过增元. 纳米通道滑移流动的分子动力学研究. 工程热物理学报, 2003, 24: 670-672

10 王慧, 胡元中, 邹鲲, 等. 纳米摩擦学的分子动力学模拟研究. 中国科学(A 辑), 2001, 44: 1049-1055

11 曾凡林, 孙毅. 纳米薄膜润滑及其改性的分子动力学模拟. 机械工程学报, 2006, 42: 138-143

12 Bao F B, Lin J Z, Shi X. Burnett simulations of flow and heat transfer in micro couette flow using second-order slip conditions. Heat Mass Transfer, 2007, 43: 559-566

13 Bao F B, Lin J Z. Burnett simulation of gas flow and heat transfer in micro poiseuille flow. Int J Heat Mass Transfer, 2008, 51: 41394144

14 Bao F B, Lin J Z. Burnett simulations of gas flow in microchannels. Fluid Dyn Res, 2008, 40: 679-694

15 Wu Y H, Wiwatanapataphee B, Hu M B. Pressure-driven transient flows of Newtonian fluids through microtubes with slip boundary. Physica A, 2008, 387: 5979-5990

16 袁斌, 张国贤, 吴白羽, 等. 径向滑动轴承边界滑移现象研究. 润滑与密封, 2001, 2: 29-30, 42

17 缪协兴, 王钦方, 陈旭昌, 等. 润滑脂在钢管中流动的壁滑移实验和研究. 哈尔滨工业大学学报, 2007, 39: 1172-1176

18 Zhou H W, Zhang Y H, Li A M, et al. Experimental study on moving boundaries of fluid flow in porous media. Chinese Sci Bull, 2008, 53: $2438-2445$

19 Wu C W, Ma G J. On the boundary slip of fluid flow. Sci China Ser G: Phys Mech Astron, 2005, 48: 178-187

20 田文超, 贾建援. 微尺度连续介质法定义域分析. 计算力学学报, 2005, 22: 189-192

21 田文超，贾建援. Hamaker 均质材料假设修正. 物理学报, 2003, 52: 1061-1065

22 Dedkov G V, Dyshekov M B. Deformation of the contact region and adhesional friction between nanoprobe and surface. Surf Sci, 2000, 463: $125-134$

23 Kirkwood J G. The statistical mechanical theory of transport processes I. General theory. J Chem Phys, 1946, 14: 180-201

24 Rice S A, Kirkwood J G. On an approximate theory of transport in dense media. J Chem Phys, 1959, 31: 901—908

25 尤学一, 郑湘君, 李丹. 微尺度流道内液体摩擦系数的理论计算. 中国机械工程, 2005, 16: 1278-1281 